\title{
Cryogenic design for a high intensity ultracold neutron source at TRIUMF
}

\author{
Shinsuke Kawasaki ${ }^{\mathrm{a}}$ and Takahiro Okamura (TUCAN collaboration)
}

KEK, Oho 1-1, Tsukuba, Ibaraki, Japan

\begin{abstract}
The TUCAN (TRIUMF Ultra-Cold Advanced Neutron) collaboration has been developing a source of high-intensity ultra-cold neutrons for use in a neutron electric dipole search. The source is composed of a spallation neutron source and a superfluid helium ultra-cold neutron converter, surrounded by a cold moderator. The temperature of the superfluid helium needs to be maintained at approximately $1.0 \mathrm{~K}$ to suppress up-scattering by phonons. The Kapitza conductance and the heat transport by the superfluid helium are key parameters which need to be well characterized. We have therefore investigated them in first experiments. Current efforts are directed at optimizing the design of the helium cryostat.
\end{abstract}

\section{Introduction}

Ultra-cold Neutrons (UCNs) are extremely slow neutrons with kinetic energies below several hundred neV. As a consequence, UCNs are totally reflected at the surface of certain materials and can be confined in a material bottle. Using this unique property, UCNs are employed in various experiments such as neutron electric dipole moment (nEDM) searches, neutron lifetime measurements, and gravity experiments. Improvement of the statistical uncertainty of these experiments requires a high-intensity UCN source.

The TUCAN (TRIUMF Ultra-Cold Advanced Neutron) collaboration has been developing a high-intensity UCN source on a dedicated proton beam line at TRIUMF. A flagship experiment is an nEDM search with the aim of achieving $10^{-27} \mathrm{ecm}$ sensitivity. The current upper limit on the neutron EDM is $3.0 \times 10^{-26} \mathrm{ecm}$ [1] where UCN statistics was a dominating limitation of the sensitivity.

Our UCN source is composed of a spallation neutron source and a superfluid helium UCN converter. The prototype UCN source, which was developed at the Research Center for Nuclear Physics, Osaka, Japan [2], was transported to TRIUMF where it was installed. Commissioning of this source commenced at TRIUMF in November 2017. Following first successful UCN production, a significant upgrade is planned with the goal to obtain the highest UCN intensity in the world. Key component of the improved apparatus is a new helium cryostat to remove high radiation heat from the superfluid helium UCN converter and thus allow for higher proton beam currents on the spallation target. Furthermore, the intention is to change the cold moderator from solid heavy water to liquid deuterium in order to obtain a higher cold neutron flux at the location of the converter.

\footnotetext{
a e-mail: shinsuke.kawasaki@kek.jp
}

\section{TUCAN source}

A dedicated proton beam line for the UCN source was constructed in the meson hall at TRIUMF. The beam line can supply a $480 \mathrm{MeV} \times 40 \mu \mathrm{A}$ proton beam on a tungsten target. High-energy neutrons are produced by spallation reaction and slowed down to the cold-neutron energy range by neutron moderators consisting of heavy water and liquid deuterium. The cold neutrons excite phonons in the superfluid-helium converter whereby they lose almost all their kinetic energy and thus become UCNs.

Figure 1 presents a schematic diagram of the TUCAN source. The superfluid helium UCN converter of 21 liters is surrounded by 125 liters of liquid deuterium. A study using the particle simulation tool MCNP6 [3] has been conducted to optimize UCN production. This study estimated the heat load on the superfluid helium and its vessel to be $5-10 \mathrm{~W}$. Further, it is necessary to maintain the superfluid helium at approximately $1.0 \mathrm{~K}$, because at this temperature the UCN storage lifetime due to phonon up-scattering is about $100 \mathrm{~s}$ and deceases with increasing temperature of the superfluid helium in proportion to $T^{-7}$ [4]. This heat is transported to the helium cryostat by a $1.5 \mathrm{~m}$ long UCN guide filled with superfluid helium as shown in Fig. 1.

\section{Helium cryostat}

The superfluid helium converter is cooled by decompressed liquid ${ }^{3} \mathrm{He}$ via a heat exchanger. ${ }^{3} \mathrm{He}$ is better suited than ${ }^{4} \mathrm{He}$ to achieve a liquid temperature of approximately $1.0 \mathrm{~K}$ because of its higher saturated vapor pressure. At a pumping speed of $8,000 \mathrm{~m}^{3} /$ hour, the cooling power of the pumped ${ }^{3} \mathrm{He}$ is approximately $10 \mathrm{~W}$ at a ${ }^{3} \mathrm{He}$ temperature of $0.8 \mathrm{~K}$. The helium cryostat needs to be located behind a radiation shield to prevent it from being adversely affected by activation.

(C) The Authors, published by EDP Sciences. This is an Open Access article distributed under the terms of the Creative Commons Attribution License 4.0 (http://creativecommons.org/licenses/by/4.0/). 


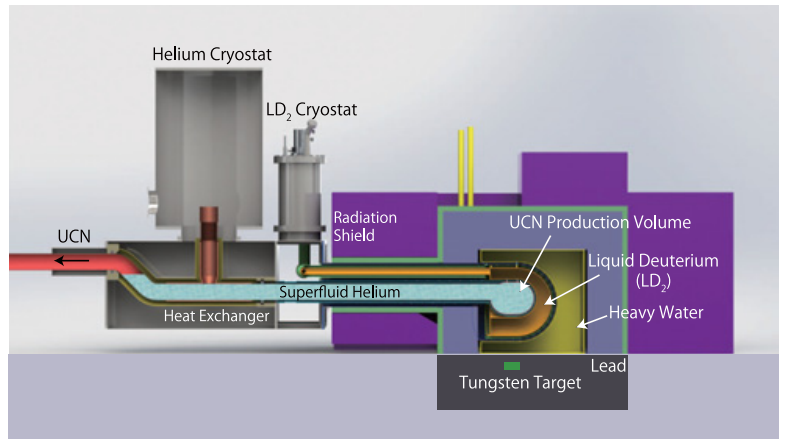

Figure 1. TUCAN source. Superfluid helium UCN converter surrounded by heavy water and liquid deuterium neutron moderators located above the spallation target. The superfluid helium is cooled by the helium cryostat.

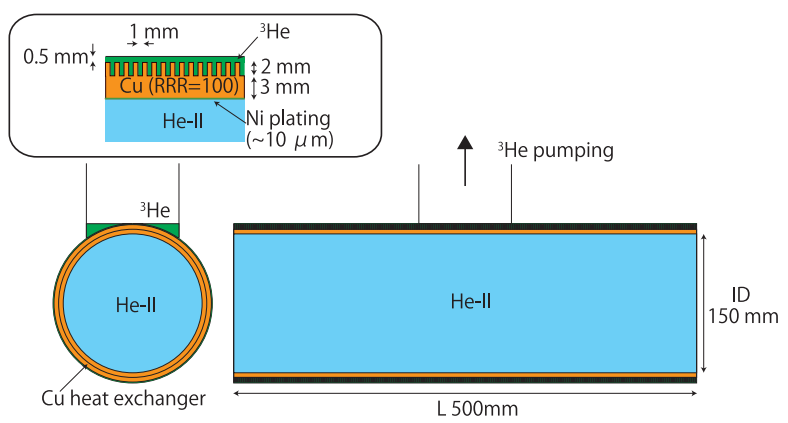

Figure 2. Design of the heat exchanger. The interior is filled with superfluid helium and is surrounded by an exterior layer filled with ${ }^{3} \mathrm{He}$.

In this system, temperature gradients mainly occur at the heat exchanger and in the superfluid helium of the UCN guide.

\subsection{Heat exchanger}

A cylindrical heat exchanger, manufactured from oxygenfree copper, is used to remove the heat produced in the converter. As shown in Fig. 2, the interior of the heat exchanger is filled with the superfluid helium for neutron conversion, while its external surface is in contact with liquid ${ }^{3} \mathrm{He}$. Fins on the outer surface increase the contact area with the ${ }^{3} \mathrm{He}$. On the other hand, the inner surface of the heat exchanger is kept flat to avoid excessive UCN wall losses that would be associated with a fin structure. In addition, the it is plated with nickel phosphorus (NiP) to increase its Fermi potential.

\subsection{Kapitza conductance}

The Kapitza conductance $h_{K}$, as discussed in several text books (see, e.g., Ref. [5]), is the conductance at the thermal boundary between a solid and liquid helium;

$$
q=h_{K}\left(T_{S}-T_{L}\right),
$$

where $q$ is the heat flux and $T_{S}$ and $T_{L}$ are temperature of the solid and the liquid, respectively.

This quantity is predicted by two theories. The phonon radiation limit describes the Kapitza conductance as

$$
h_{K}=\frac{4 \pi^{5} k_{B}^{2}}{5 \Theta_{D}^{2} h}\left(\frac{3 n}{4 \pi}\right)^{2 / 3} T^{3},
$$

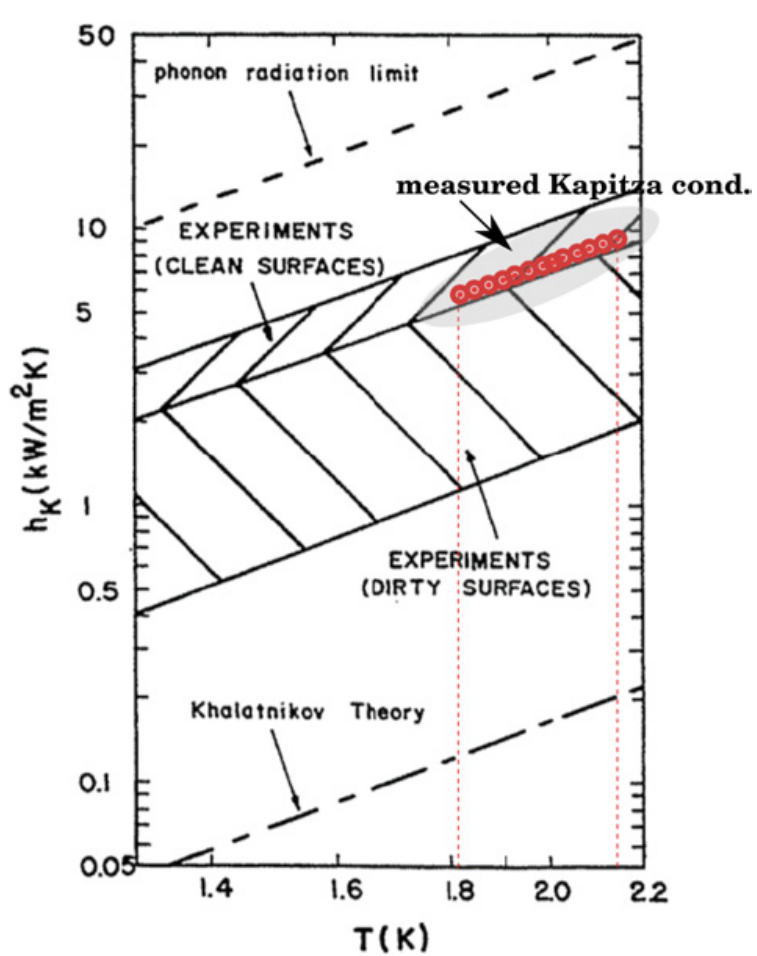

Figure 3. Kapitza conductance between copper and superfluid helium. The conductance is affected by surface cleanliness. The picture is taken from [5] (Fig. 5.22). The filled red circles show the result of our measurement at KEK (this work).

where $\Theta_{D}$ is the Debye temperature, $n$ is the number density of atoms of the solid, and $k_{B}$ and $h$ are Boltzmann's and Planck's constants, respectively.

On the other hand, the Khalatnikov theory is based on an analogy with classical acoustics or boundary scattering in optics and leads to

$$
h_{K}=\frac{2 \pi^{2} \rho_{L} c_{L} k_{B}^{4}}{15 \hbar^{3} \rho_{S} v_{t}^{3}} F T^{3},
$$

where $\rho_{L}$ and $\rho_{S}$ are the densities of the liquid and solid, respectively, $c_{L}$ is the sonic velocity in the superfluid helium, $v_{t}$ is the transverse component of the sound velocity in the solid, and $F$ is the solid angle integration with respect to the transmission and reflection of phonons at the solid-liquid interface. $F$ strongly depends on the velocity of sound in the solid. For our case of a copper${ }^{4}$ He interface $F$ can be taken as 1.5 [5].

Figure 3 shows values derived from the two theoretical models, together with actual experimental data of the Kapitza conductance between copper and superfluid helium [5]. In both theories it is proportional to $T^{3}$, however, with a large discrepancy in the predicted absolute values. These do not agree with the experimental data which lie in between. To normalize the coefficient to experimental results, a correction factor $K_{G}$ is used:

$$
h_{K}=K_{G} \times 20 \frac{\mathrm{W}}{\mathrm{m}^{2} \mathrm{~K}^{4}} \times T^{3} .
$$

As discused in Ref. [5], a value of $K_{G}=225$ represents the prediction of the phonon radiation limit theory, and $K_{G}=1$ of the Khalatnikov theory. The surface cleanliness of the copper affects the Kapitza conductance, which can be increased by mechanical and chemical treatments. 
Table 1. Heat exchanger parameters.

\begin{tabular}{ll}
\hline Material & oxygen-free copper $(\mathrm{RRR}>100)$ \\
Length & $500 \mathrm{~mm}$ \\
Diameter & $150 \mathrm{~mm}$ \\
Fin length & $2 \mathrm{~mm}$ \\
Fin pitch & $1 \mathrm{~mm}$ \\
Surface area: inside & $0.24 \mathrm{~m}^{2}$ \\
outside & $0.76 \mathrm{~m}^{2}$ \\
\hline
\end{tabular}

Empirically, a $K_{G}$ value between 6.5 and 65 is obtained for various surface conditions.

The Kapitza conductance between copper and ${ }^{3} \mathrm{He}$, which is a fermion, is estimated by the following equation. Its form is very similar to that of the Eq. (3), which is satisfied in the case of the Bose system ${ }^{4} \mathrm{He}$ :

$$
h_{K(3 \mathrm{He})}=\frac{2 \pi^{2} a_{1} \rho_{L} c_{0} k_{B}^{4}}{15 \hbar^{3} \rho_{S} v_{t}^{3}} F T^{3} \text {. }
$$

This equation was derived based on the Fermi-liquid theory [6]. According to [7], for $a_{1} \simeq 1.54$, the zero sound velocity becomes $c_{0}=3.456 v_{f}$. Here, $v_{f}$ is the Fermi velocity. A practical range of values is becomes $v_{f}=1.8-3.9 \times 10^{3} \mathrm{~cm} / \mathrm{sec}$, valid for various pressure conditions. Considering the different densities and sound velocities of ${ }^{3} \mathrm{He}$ and superfluid helium, the Kapitza conductance between copper and ${ }^{3} \mathrm{He}$ is of the same order as that between copper and superfluid helium:

$$
h_{K(\mathrm{He}-\mathrm{II})}=a \times h_{K(3 \mathrm{He})} \quad a=1.2-2.6
$$

The Kapitza conductance between nickel and superfluid helium is estimated with Eq. (2). Comparing the Debye temperatures ( $\mathrm{Cu}: 343.5 \mathrm{~K}$ and Ni: $450 \mathrm{~K}$ ) and atom number densities of copper and nickel, the ratio of the Kapitza conductance of these materials is

$$
h_{K(\mathrm{Ni})}=0.61 \times h_{K(\mathrm{Cu})} .
$$

Using the surface areas of the heat exchanger provided in Table 1, its Kapitza conductance can be calculated with Eqs. (6) and (7). Figure 4 shows the temperature increment at the heat exchanger as a function of temperature for various $K_{G}$ values, for a heat load $Q$ of $10 \mathrm{~W}$ and a ${ }^{3} \mathrm{He}$ temperature $T_{3 \mathrm{He}}$ of $0.8 \mathrm{~K}$. The temperature $T_{\mathrm{HEX}}$ at the outer surface of the heat exchanger is $0.97 \mathrm{~K}$ and the temperature $T_{\mathrm{He}-\mathrm{II}}$ of the superfluid helium close to the inner surface is $1.16 \mathrm{~K}$ for $K_{G}$ of 20 , and $a=2.6$, which are conservative estimatations. The temperature difference along the fins and in the bulk of the heat exchanger can be neglected, because heat conductance in the bulk is much larger than the Kapitza conductance.

The temperature increase by the Kapitza conductance of the heat exchanger strongly depends on the value of $K_{G}$. A total temperature increase below $0.3 \mathrm{~K}$ across both surfaces requires a value of $K_{G}$ larger than 25 for a heat load of $10 \mathrm{~W}$.

The Kapitza conductance largely depends on the surface properties of the heat exchanger as shown in Fig. 3; thus, the conductance should be measured before fabricating the actual heat exchanger. Figure 5 shows a sample that was used to measure it. A polyimide
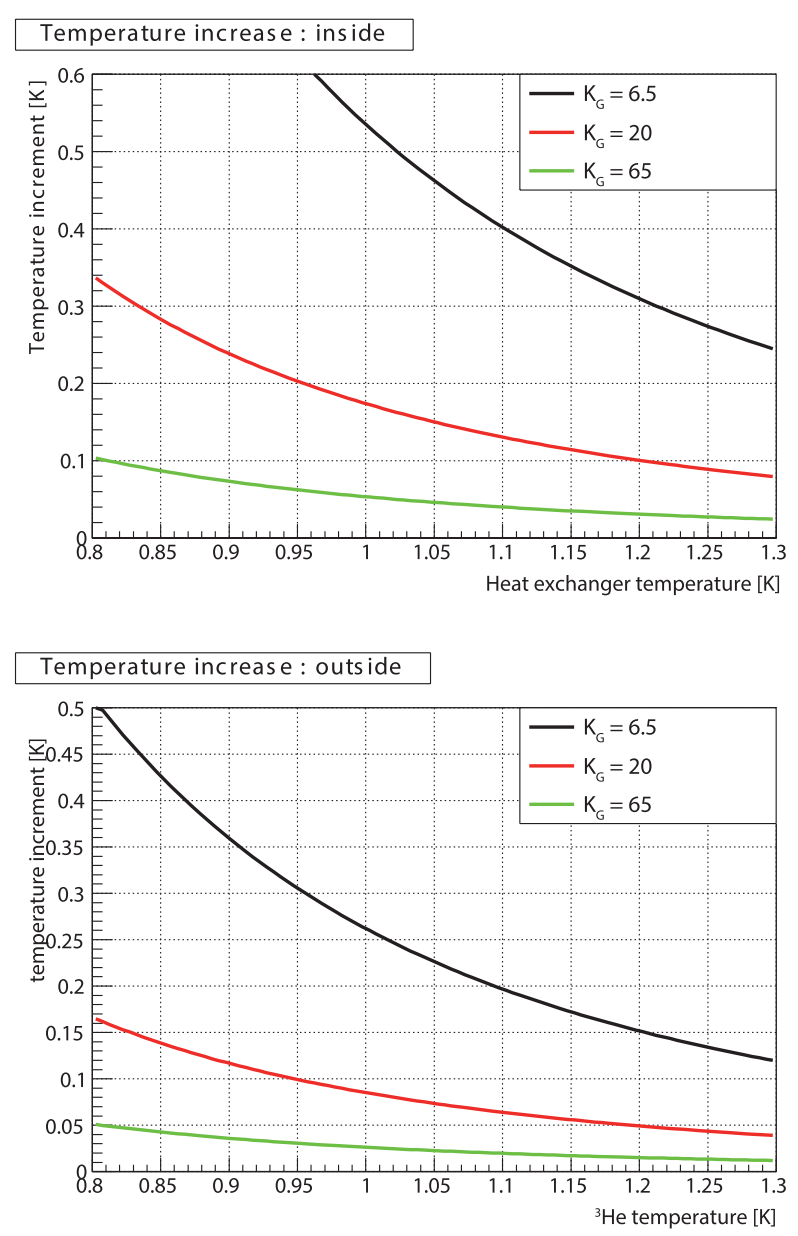

Figure 4. Temperature difference across liquid-solid interface owing to the Kapitza conductance at the heat exchanger for a $10-\mathrm{W}$ heat load, inside (top), outside (bottom). Values are calculated using $a=2.6$ (see Eq. (6)).

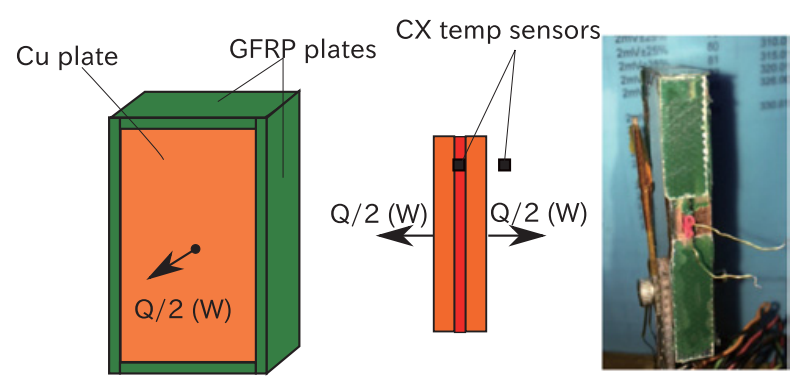

Figure 5. Experimental setup to measure the Kapitza conductance.

thermalfoil heater with surface area $A=45 \mathrm{~mm} \times 25 \mathrm{~mm}$ is inserted between two copper plates of the same size. Its heat $Q_{\text {in }}$ generates a heat flux of

$$
q_{\text {in }} \simeq \frac{Q_{\text {in }} / 2}{A}
$$

across each copper plate. To prevent heat escaping through the side walls, they are covered by GFRP insulator. Two Cernox temperature sensors are employed. The first is situated between the copper plates and the second is used to measure the temperature of the superfluid helium. The Kapitza conductance can then be determined from the temperature difference between the two temperature 


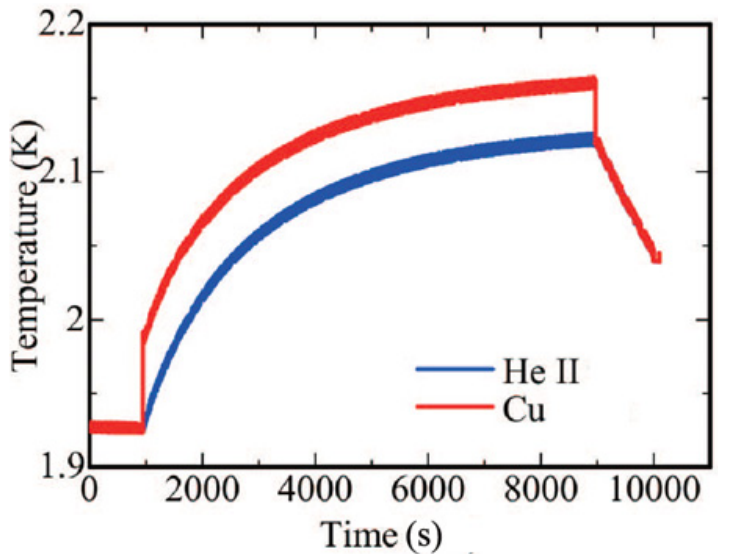

Figure 6. Result of Kapitza conductance measurement in the temperature range $1.93 \mathrm{~K}$ to $2.15 \mathrm{~K}$.

sensors $\Delta T=T_{\mathrm{Cu}}-T_{\mathrm{He} \text { II }}$ and the heat flux $q_{\text {in }}$ from Eq. (8):

$$
h_{K}=\frac{q_{\text {in }}}{\Delta T} .
$$

Measurements were performed using standard oxygenfree high-conductivity copper with a purity of $99.99 \%$. The measurements were done for the temperature range from $1.85 \mathrm{~K}$ to $2.15 \mathrm{~K}$ since the cooling power of an available cryostat was limited. Figure 6 shows a result for $1.95 \mathrm{~K}$ to $2.15 \mathrm{~K}$. The temperature difference between the copper sample and the superfluid helium was measured for two different heat inputs, $0.5 \mathrm{~W}$ and $0.8 \mathrm{~W}$. During the measurement, the temperature of the superfluid helium increased very slowly; therefore, the system can be assumed to be quasi-static. Figure 3 shows that the measured values we obtained for the Kapitza conductance follow the expected $T^{3}$ dependence, and yield a value of $K_{G}$ between 45 and 48 (which is larger than the conservative value of 20 assumed in the estimate of heat exchanger efficiency given above).

It should however be noted that different materials have different Kapitza conductances. In our work we selected to use $\mathrm{NiP}$ as the plating on the inner surface of the heat exchanger to increase its Fermi potential. Before fabrication of the heat exchanger we plan to measure the Kapitza conductance of NiP-plated copper samples in a range of lower temperatures.

\subsection{Heat transfer in superfluid helium}

Here we analyze the heat transport through the superfluidhelium filled UCN guide between the UCN source chamber and the heat exchanger. The well known twofluid model can often be used to describe the thermo-fluid behavior of superfluid helium. The superfluid component can be regarded as a Bose-Einstein condensate with no entropy and no viscosity. The normal fluid component contains the thermally excited quasi-particles, such as phonons and rotons, and has both entropy and viscosity. Therefore, only the normal fluid component plays a role in energy transport. The lower is the temperature, the smaller is the normal fluid component in superfluid helium.

According to two-fluid dynamics, heat transfer in a superfluid occurs via a counter flow between the superfluid and normal fluid components. If the relative velocity

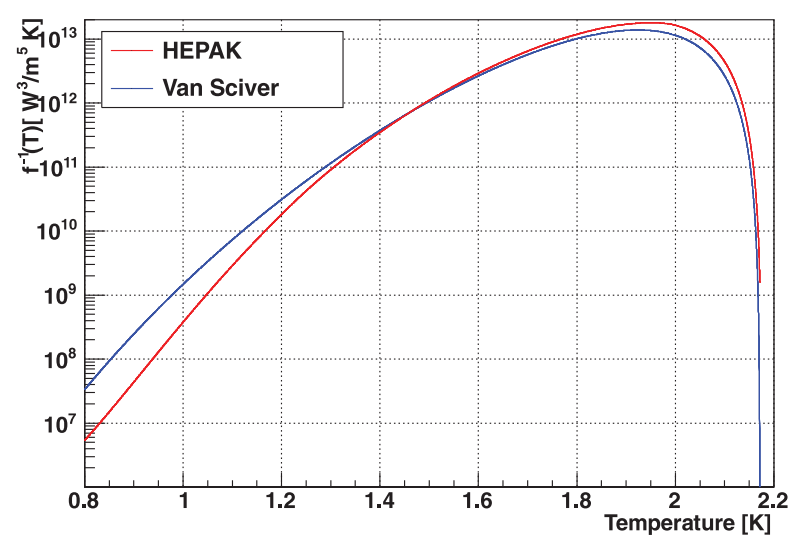

Figure 7. $f(T)^{-1}$ as a function of temperature. The red line is derived from HEPAK [9] and the blue line from Van Sciver [5] .

between these two components exceeds a critical value, mutual friction occurs between them. This is regarded as a superfluid turbulent state. The Reynolds number of the normal fluid component is described as

$$
R e_{n}=\frac{u_{n} D_{\mathrm{UCN}}}{v_{n}}, \quad v_{n}=\eta_{n} / \rho,
$$

where $u_{n}$ is the velocity of the normal fluid, $\eta_{n}$ is its viscosity, $\rho$ is the total helium density [5], and $D_{\mathrm{UCN}}$ is the UCN guide diameter. For a heat load of $10 \mathrm{~W}$ and $D_{\mathrm{UCN}}=150 \mathrm{~mm}$, the Reynolds number is calculated to be $R e_{n} \sim 7 \times 10^{5}$, using data from the HEPAK dataset [9], which provides the physical properties of helium including those in the superfluid regime. This is much larger than the critical value $R e_{n c}=1200$. Therefore, the flow can be regarded as turbulent superfluid.

The temperature gradient during turbulent heat transport in steady-state can be described with

$$
\frac{d T}{d x}=-f(T) q^{3}
$$

where $f(T)=A_{G M} \rho_{n} /\left(\rho_{s}^{3} s^{4} T^{3}\right)$ is a heat conductivity function. $A_{G M}$ is the Gorter-Mellink mutual friction parameter, $s$ is the entropy, and $\rho_{n}$ and $\rho_{s}$ are the normal and superfluid densities. The values of $A_{G M}$ are also available from HEPAK [9].

Equation (11) can be written as

$$
q=-\left(f(T)^{-1} \frac{\delta T}{\delta x}\right)^{1 / 3}
$$

Based on an empirical fit by Van Sciver [5] to the Gorter-Mellink parameter, it is possible to parametrize this heat conductivity function:

$$
f^{-1}(T)=g\left(T_{\lambda}\right)\left[t^{5.7}\left(1-t^{5.7}\right)\right]^{3},
$$

where $g\left(T_{\lambda}\right)=\rho^{2} s_{\lambda}^{4} T_{\lambda}^{3} / A_{\lambda}, t=T / T_{\lambda}, s_{\lambda}=1.559 \mathrm{~J} /(\mathrm{kg} \cdot \mathrm{K})$, $A_{\lambda}=1450 \mathrm{~ms} / \mathrm{kg}, \quad \rho=145.2 \mathrm{~kg} / \mathrm{m}^{3}, \quad$ and $T_{\lambda}=$ $2.172 \mathrm{~K}[5]$.

Figure 7 shows the values of $f^{-1}(T)$ derived from HEPAK and Eq. (13) as a function of temperature. These values have been determined with high accuracy by many experiments at temperatures around $1.4 \mathrm{~K}$ or more. However, as shown in the figure, a model dependency can 


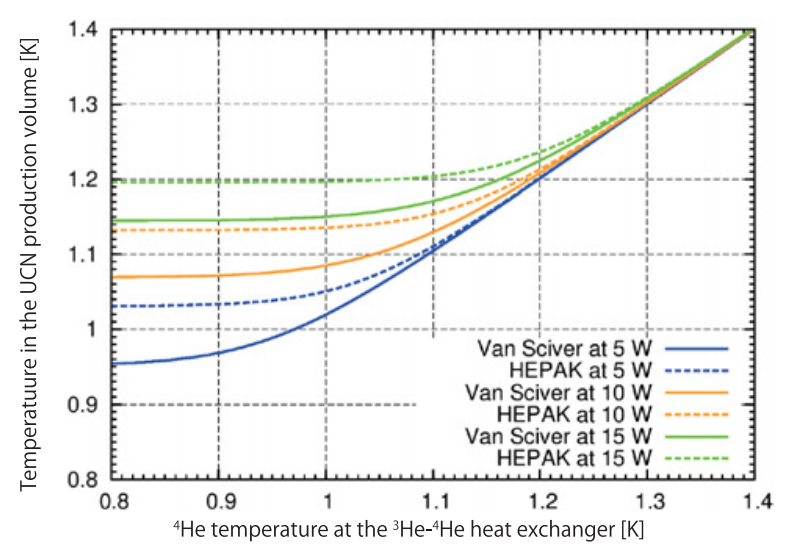

Figure 8. Temperature in the UCN production volume as a function of the superfluid-helium temperature at the ${ }^{3} \mathrm{He}-{ }^{4} \mathrm{He}$ heat exchanger, for various heat loads using $f(T)^{-1}$ derived from Van Sciver [5] and HEPAK [9]. The UCN guide diameter is $150 \mathrm{~mm}$ and its length is $1.5 \mathrm{~m}$.

be seen at temperatures below $1.4 \mathrm{~K}$. There is little data on the heat transfer function in the superfluid turbulent region at larger diameters because it is quite difficult to reach such low temperatures without using ${ }^{3} \mathrm{He}$. We plan to measure the value of $f(T)^{-1}$ below $1.4 \mathrm{~K}$ by additional tests.

The temperature difference between the $\mathrm{UCN}$ prodution volume and the helium cryostat can be calculated using $f(T)^{-1}$. In a straight channel with a length $L$ and a cross section $A$, the total heat transport $Q$ is given by

$$
Q=\left(\frac{A^{3}}{L} \int_{T_{L}}^{T_{H}} f(T)^{-1} d T\right)^{1 / 3}
$$

where $T_{L}$ and $T_{H}$ are low-temperature and hightemperature end of the superfluid-helium. Figure 8 shows the temperature at both ends, calculated for $A=D_{U C N}=$ $150 \mathrm{~mm}$ and $L=1.5 \mathrm{~m}$, and for different heat loads in the UCN production chamber.

Table 2 lists the temperatures at the heat exchanger and in the UCN source chamber, for values of the function $f(T)^{-1}$ derived from the HEPAK and Van Sciver equations. The correction factor $K_{G}$ of the Kapitza conductance (see Eq. (4)) typically exceeds 20. For $K_{G}>40$, as indicated by our latest measurements, we can thus achieve a UCN source temperature below $1.14 \mathrm{~K}$.
Table 2. Important temperatures of the UCN source cryostat. The conversion factor $a$ (see Eq. (6) is assumed to be 2.6 .

\begin{tabular}{c|c|cc|cc}
\hline \multirow{2}{*}{$\begin{array}{c}\text { heat } \\
\text { load }\end{array}$} & \multirow{3}{*}{$\mathrm{3} \mathrm{He}$} & \multicolumn{3}{|c}{ Temperature [K] } \\
\cline { 3 - 6 } & & \multicolumn{2}{|c}{$K_{\mathrm{He}} \mathrm{HEX}$} & \multicolumn{2}{c}{$T_{\text {prod }}$} \\
\hline \multirow{2}{*}{$10 \mathrm{~W}$} & \multirow{2}{*}{0.8} & 1.16 & 40 & \multicolumn{2}{|c}{$f(T)^{-1}$} \\
& & & 1.00 & 1.17 & 1.18 \\
\hline
\end{tabular}

\section{Summary}

The TUCAN collaboration has been developing a highintensity UCN source at TRIUMF. This source combines a spallation neutron source and a superfluid helium $\mathrm{UCN}$ converter. Effective UCN production requires the superfluid temperature to be maintained at approximately $1.0 \mathrm{~K}$. The heat load from the spallation target is estimated to be $10 \mathrm{~W}$. The development of the helium cryostat to remove this amount of heat is ongoing. In this article we have analyzed in some detail the Kapitza conductance and the heat transfer in superfluid helium, which are key parameters for achieving the target temperature. The latest measurement of the Kapitza conductance showed that the thermal conductance of the heat exchanger is sufficient. Further experiments are planned to finalize the design of the helium cryostat.

This work was supported by JSPS KAKENHI Grant Numbers JP17K14307, JP18H03702 and JP18H05230.

\section{References}

[1] J.M. Pendlebury et al., Phys. Rev. D 92, 092003 (2015)

[2] Y. Masuda et al., Phys. Rev. Lett. 108, 134801 (2012)

[3] T. Goorley et al., Nucl. Technol. 180, 298 (2012)

[4] K.K.H. Leung, S. Ivanov, F.M. Piegsa, M. Simson, O. Zimmer, Phys. Rev. C 93, 025501 (2016)

[5] S.W. Van Sciver, Helium cryogenics (Springer Science \& Business Media, 2012)

[6] G.L. Pollack, Rev. Mod. Phys. 41, 48 (1969)

[7] N. Nishiguchi, T. Nakayama, Phys. Rev. B 25, 5720 (1982)

[8] M. Sciacca, L. Galantucci, Phys. Lett. A 378, 2471 (2014)

[9] Cryodata. Inc., HEPAK User's Guide (Horizon Technologies, 2005) 